\title{
Band Structures of Edge Magnetoplasmon Crystals
}

\author{
Ken-ichi Sasaki* \\ NTT Research Center for Theoretical Quantum \\ Physics and NTT Basic Research Laboratories, \\ NTT Corporation, 3-1 Morinosato Wakamiya, \\ Atsugi, Kanagawa 243-0198, Japan
}

(Dated: December 3, 2021)

\begin{abstract}
A two-dimensional electron gas in a static external magnetic field exhibits two distinct collective excitation modes. The lower frequency mode propagates along the periphery of the domain almost freely with an extended lifetime, which is referred to as edge magnetoplasmons. Peculiar phenomena caused by a capacitive interaction between nearest neighbor domains are known, such as the emergence of Tomonaga-Luttinger liquid and charge density fractionalization. Meanwhile, the number of coupled domains investigated in the past has been limited to a small number. Here, we performed calculations using a continuum model of edge magnetoplasmons, the band structures of planar crystals composed of an arbitrary number of domains, including a chain, ladder, and honeycomb network, with the general interaction strength. We explain the band structures in terms of the fundamental collective modes of a molecule composed of two equivalent domains. These are the extended chiral propagation modes that yield a linear dispersion band and the standing wave modes localized in the coupled regions that cause a flat band. The chain's band structures resemble the miniband structures calculated from the Kronig-Penny model for the electron in a semiconductor superlattice. We point out that a geometrical deformation of the chain does not change the band structures as it can be expressed as a gauge degree of freedom that only causes a shift in the wavenumber.
\end{abstract}




\section{INTRODUCTION}

Many physical systems have the collective excited states, known as plasmons, in which the electrons and electromagnetic field are dynamically coupled to form a self-sustainable motion of the composites. When plasmons exist in each component (or domain), they can interact with each other through electromagnetic fields if the domains are close enough to each other. In this manuscript, we examine the characteristics of the plasmons of the entire system, as regards the basic plasmons localizing and propagating along the edge of each planar domain, which are referred to as the edge magnetoplasmons (EMPs) $\underline{\underline{1}} \underline{\underline{3}}$ EMPs are the low-energy excited states of a planar system of a two-dimensional electron gas in a stationary external magnetic field applied perpendicular to the plane, and they exhibit a chiral propagation that moves in a direction determined by the orientation of the magnetic field. It is known that EMPs exhibit peculiar phenomena caused by a capacitive interaction between nearest neighbor domains, such as the emergence of Tomonaga-Luttinger liquid

and charge density fractionalization. $\underline{\underline{4}}$ There is also a theoretical proposal that EMPs are potential candidates for quantum energy teleportation, in which energy transportation can be realized by classical information without energy carriers, and the interaction between EMPs plays an essential role in it. $\frac{5}{}$

Besides the fact that experiments are scheduled and theoretical consideration is called for, we have other motivation for getting onto the subject of the EMPs in a domain network. First, if we regard a single planar domain, at the edge of which EMPs exist, as a fictitious atom, our objective is to find the energy spectrum of a plasmonic crystal, or more specifically, an EMP crystal or EMP molecule. Naturally, since a plasmon is a hybrid of electrons and photons (electromagnetic fields), such a plasmonic crystal must have an essential relationship to a photonic crystal. Indeed, we will show that there is a close similarity between an EMP crystal and semiconductor superlattice, which is a periodic structure of layers of two (or more) materials and the simplest example of a photonic crystal. When discussing the interaction between adjacent domains, the idea of static atomic orbitals, such as the bonding and anti-bonding orbitals which are useful in discussing the formation or stability of a lattice, may be extended to the chiral and dynamical counterparts. Second, we seek to gain a better understanding of the nature of EMPs in a network of domains. For example, we would like to know the lowest energy excited state of the whole system. Is it still an EMP that 
propagates along the outer edge of the whole system?

We propose in this manuscript a general method to calculate the energy spectrum of a planar EMP crystal. We first show the results for the simplest EMP molecule consisting of the two domains (Sec. III), which are applicable for any finite number of domains and essential to understand the physics. Next, the dispersion relation of the plasmons in the periodic system consisting of $N$ domains, namely the energy band structures of the periodic EMP crystal, is analytically constructed for a chain, ladder, and honeycomb network (Sec. IV). We will show that the naive "EMP" of the EMP crystal, which has the same chirality as the atomic EMP, is not the lowest energy state of the entire system for the general interaction strength. We also discuss an extension of the planar EMP crystals toward three-dimensional counterparts, which is useful in identifying the topological aspect of a system.

\section{BASIC KNOWLEDGE ABOUT EMP IN A SINGLE DOMAIN}

In this section, we review the two main properties of an EMP in a single domain and introduce an effective model used for our analysis.

\section{A. Two Main Properties of EMP}

First, an EMP pulse propagates almost freely along the edge of a two-dimensional electron gas in the direction determined by the magnetic-field orientation. $\frac{6}{}$ This suggests that the dispersion relation of EMPs is approximately linear. Indeed, when the boundary potential is sufficiently sharp so that the electron density changes abruptly, Volkov and Mikhailov solved an integral equation of the electric potential with the Wiener-Hopf method under reasonable assumptions and succeeded in getting the dispersion relation $\operatorname{as}^{7.8}$

$$
\omega\left(q_{y}\right)=\frac{2 q_{y} \sigma_{x y}}{\kappa}\left(\ln \frac{2}{\left|q_{y}\right| \ell_{x}}+1\right),
$$

where $q_{y}$ is the wavevector along the edge, $\sigma_{x y}$ is the static Hall conductivity, $\kappa$ is the relative dielectric constant, and $\ell_{x}$ is the localization length of the charge density (in the direction perpendicular to the edge), which is proportional to the dynamical conductivity $\sigma_{x x}(\omega)$ :

$$
\ell_{x}=\frac{2 \pi i \sigma_{x x}(\omega)}{\omega \kappa}
$$


Since $\ell_{x}$ may depend on $\omega$, the EMP frequency is determined by solving Eqs. (11) and (2) self-consistently. Practically, $\ell_{x}$ is independent of $\omega$ as $\ell_{x} \simeq \frac{e^{2} \nu}{\kappa \hbar \omega_{c}}$ (where $\nu$ is the filling factor, and $\omega_{c}$ is the cyclotron frequency), because $\sigma_{x x}(\omega) \simeq-i \frac{\omega}{2 \pi} \frac{e^{2} \nu}{\hbar \omega_{c}}$ holds and $\omega$ in the numerator of Eq. (2) is canceled out by that of the denominator. The wavelength of interest is usually much larger than $\ell_{x}$, which makes the dispersion relation of EMPs approximately linear. The linear chiral dispersion is in sharp contrast to the gapped spectrum of bulk magnetoplasmons (MPs), which is written in terms of $\omega_{c}$ and two-dimensional plasmon frequency $\omega_{p}$ as $\sqrt{\omega_{p}^{2}+\omega_{c}^{2}}$.

Second, the EMP damping is suppressed by the applied magnetic field. If damping is significant, EMPs would not be observed in a strong magnetic field, because $\sigma_{x y}$ makes the frequency lower and lower by increasing the magnetic field and the Drude peak may obscure the EMP signal. The origin of the long EMP lifetime is a subtle problem. In a previous paper,$\underline{9}$ we argued that an internal magnetic field was neglected in theoretical approaches ${ }^{7}$ and that this simplification prevented the EMP lifetime from being determined. On the other hand, we found that the following approximate relationship between the EMP lifetime and MPs exists:

$$
\tau_{\mathrm{emp}}=\frac{\omega_{c}^{2}+\omega_{p}^{2}}{\omega_{p}^{2}} \tau_{\mathrm{mp}}
$$

This result is obtained by noticing that peculiar plasmons whose frequencies are purely imaginary exist in the interior of a two-dimensional electron gas described by the Drude model. $\stackrel{10}{ }$ When an external magnetic field is applied to the system, these bulk plasmons are still non-oscillating and are isolated from the MP. They are mainly in a transverse magnetic mode and can combine with a transverse electronic mode locally at an edge of the system to form EMPs. We note that though Eq. (3) reasonably explains experimental results, $\frac{11}{1}$ the derivation is classical, and that whether it can be extended to the quantum Hall regime is unknown.

The quantum Hall effect (QHE) is not the necessary condition for the existence of EMPs, but EMP lifetime is elongated by the QHE. The QH state is characterized by an electronic ground state whose excitation spectrum is gapful, an incompressible liquid state, in the bulk but is gapless at the edge. The energy spectrum of the QHE is similar to that of MPs, namely an MP is gapful, but an EMP is gapless. The dynamical aspect of the edge states in fractional QHEs, where interactions between electrons contribute to an incompressible 
state, has been explored by many authors. $\underline{12}-18$

\section{B. One-dimensional effective model}

The results for a single domain presented above, which are based on the classical field theory of electrodynamics, are essential and very useful in understanding experimental results. However, they are difficult to extend to more complicated physical circumstances in which EMPs interact with each other. The presence of $\kappa$ in Eq. (1) already suggests that the propagation velocity of EMPs depends on the system parameters, including its environments. $\frac{19}{}$ Hashisaka et al ${ }^{20}$ proposed a distributed-element circuit model of interacting EMPs, which introduces a geometrical capacitance $c_{x}$ for mutual interactions in addition to a channel capacitance that simulates the propagation velocity of an isolated EMP as

$$
v=\frac{\sigma_{x y}}{c_{c h}}
$$

This model is plausible and capable of describing the capacitive interactions between counterpropagating EMPs, $\stackrel{20,21}{2}$ as well as those between co-propagating EMPs. $\underline{4}^{22}$ In the coupled region of the two domains, the chiral nature of the EMPs in each domain is disturbed by $c_{x}$, and the mixed mode is formed as a non-chiral standing wave. The model can be extended to include the effect of a general type of gate needed to control the carrier density or the velocity. ${ }^{19}$ We therefore adopt the model in calculating the energy spectrum of coupled domains.

\section{TWO DOMAINS}

We assume that when the interaction between the two domains is negligibly small, the EMP pulses [expressed by humps in Fig. [1(a)] can propagates independently along the edge of each domain with velocity $v$ and without any dissipation. We will neglect the complicated EMP profile in the direction perpendicular to the edge and focus on the dynamics along the edge. The EMP dynamics of the first domain is expressed by the normal modes of the current and charge densities, $j_{1}(x, t)=a v e^{i \frac{\omega}{v}(x-v t)}$ and $\rho_{1}(x, t)=a e^{i \frac{\omega}{v}(x-v t)}$, which are a function of $x-v t$ that represents the chiral character of EMPs. The charge density (at $x=v t$ ) can be positive or negative depending on the sign of $a$. A positive and negative 
current density means a positive and negative charge density, respectively, that propagates in the same direction determined by the chirality. The continuity equation expressing charge conservation in the first domain is given by $\partial_{t} \rho_{1}(x, t)+\partial_{x} j_{1}(x, t)=0$. Likewise, we define the normal modes of current and charge densities for the second domain as $j_{2}(x, t)=b v e^{i \frac{\omega}{v}(x-v t)}$ and $\rho_{2}(x, t)=b e^{i \frac{\omega}{v}(x-v t)}$. The eigenfrequencies are quantized by the periodic boundary condition as $\omega_{1}=\left(2 \pi v / L_{1}\right) n$ and $\omega_{2}=\left(2 \pi v / L_{2}\right) n$ with integer $n$, where $L_{1}$ and $L_{2}$ are circumference of the first and second domains, respectively.

(a)

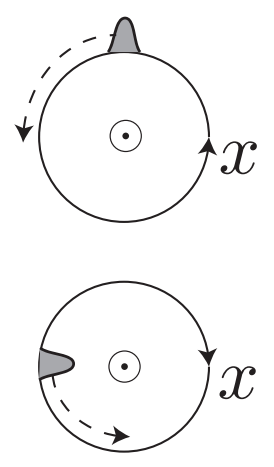

(b)

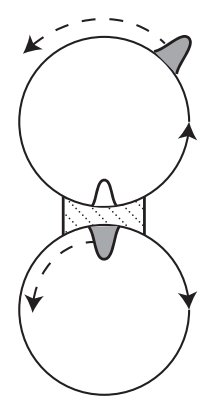

(c)

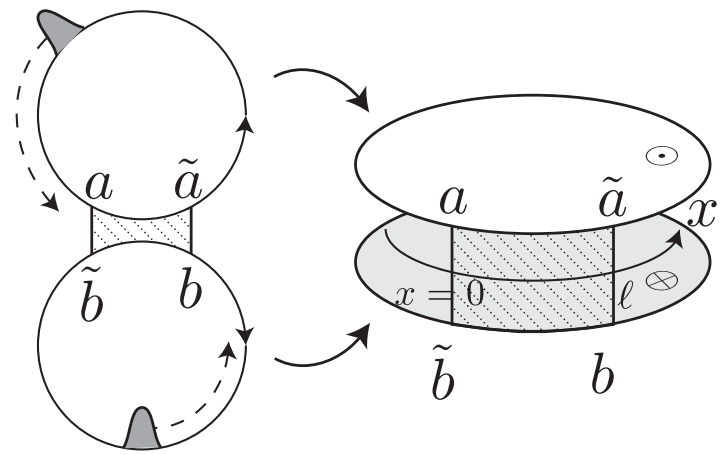

FIG. 1. Geometries of the two domains. (a) When the two domains are independent, EMPs are freely propagating along each edge. (b) When the two domains are close enough to each other, they interact with each other in the shaded part through inter-edge capacitive coupling. (c) The spatial coordinate $x$ may be shared by the two domains through the procedure discussed in the main text.

The direction of spatial coordinate $x$ is not necessarily the same (for example, anticlockwise) for the two domains. Rather, when we consider the effects of coupling between the two domains, it turns out to be convenient to define the coordinate for the second domain in the direction opposite to that for the first domain. In this new coordinate system, we have $j_{2}(x, t)=-b v e^{-i \frac{\omega}{v}(x+v t)}$ and $\rho_{2}(x, t)=b e^{-i \frac{\omega}{v}(x+v t)}$, by the replacement $v \rightarrow-v$. The minus sign is added to $j_{2}$ only (not in front of $b$ of $\rho_{2}$ ), which is necessary for them to satisfy the continuity equation $\partial_{t} \rho_{2}(x, t)+\partial_{x} j_{2}(x, t)=0$. Note that $j_{2}(x, t)$ and $\rho_{2}(x, t)$ become a function of $x+v t$, showing the same chirality as the EMPs in the first domain.

The sign difference between $j_{1}$ and $j_{2}$ (in front of $b$ ) may be explained by a fictitious procedure in three dimensions, in which the second domain is turned inside out and placed 
below (or above) the first domain, as shown in Fig. 1(c). Note that the orientation of the second domain is reversed and that the direction of a magnetic field normal to the second domain plane is reversed too. As a result, we can regard the total system as if the Hall conductivities for the two domains have different signs;

$$
\left(\begin{array}{l}
j_{1}(x) \\
j_{2}(x)
\end{array}\right)=\sigma_{x y}\left(\begin{array}{cc}
1 & 0 \\
0 & -1
\end{array}\right)\left(\begin{array}{l}
V_{1}(x) \\
V_{2}(x)
\end{array}\right)
$$

where $V_{i}(x)$ is the EMP potential given by $V_{i}(x)=\frac{\rho_{i}(x)}{c_{c h}}$. Indeed, using Eq. (4), we show that $j_{1}(x, t)=v \rho_{1}(x, t)$ and $j_{2}(x, t)=-v \rho_{2}(x, t)$, which are consistent with the normal modes. The fictitious procedure in three-dimensions makes us to notice that this system is topologically not equivalent to a capacitor in an external magnetic field (rather it is equivalent to a capacitor containing a magnetic monopole). Meanwhile, there is an EMP molecule with a staggered magnetic field that corresponds to a capacitor in a magnetic field, which is discussed in Appendix $\mathrm{A}$,

When the two domains are sufficiently close, they couple with each other through a capacitive coupling $c_{x}$ in the region $x \in[0, \ell]$ represented by shaded part between the two domains in Fig. 1(b). We assume that $c_{x}$ is a constant in the coupled region and vanishes outside. The capacitive coupling modifies charge densities through a difference between the EMP potentials of the two domains as $\rho_{1}(x)=c_{c h} V_{1}(x)+c_{x}\left(V_{1}(x)-V_{2}(x)\right)$ and $\rho_{2}(x)=c_{c h} V_{2}(x)+c_{x}\left(V_{2}(x)-V_{1}(x)\right)$. These are expressed with a $2 \times 2$ matrix as

$$
\left(\begin{array}{l}
V_{1}(x) \\
V_{2}(x)
\end{array}\right)=\frac{1}{c_{c h}}\left(\begin{array}{cc}
1-\delta & \delta \\
\delta & 1-\delta
\end{array}\right)\left(\begin{array}{l}
\rho_{1}(x) \\
\rho_{2}(x)
\end{array}\right),
$$

by defining a coupling constant

$$
\delta \equiv \frac{c_{x}}{c_{c h}+2 c_{x}}
$$

By combing Eqs. ([6) and (5), we have

$$
\left(\begin{array}{l}
j_{1}(x) \\
j_{2}(x)
\end{array}\right)=v\left(\begin{array}{cc}
1-\delta & \delta \\
-\delta & -(1-\delta)
\end{array}\right)\left(\begin{array}{l}
\rho_{1}(x) \\
\rho_{2}(x)
\end{array}\right) .
$$

Because of the continuity equation expressing independent charge conservation in each domain $\partial_{t} \rho_{i}(x, t)+\partial_{x} j_{i}(x, t)=0$, Eq. (8) becomes the following dynamical equation of the 
current density

$$
\partial_{t}\left(\begin{array}{l}
j_{1}(x, t) \\
j_{2}(x, t)
\end{array}\right)=-v\left(\begin{array}{cc}
1-\delta & \delta \\
-\delta & -(1-\delta)
\end{array}\right) \partial_{x}\left(\begin{array}{l}
j_{1}(x, t) \\
j_{2}(x, t)
\end{array}\right)
$$

The eigenvalues of the $2 \times 2$ matrix are $\pm v_{c}$, where $v_{c} \equiv v \sqrt{1-2 \delta}$ corresponds to the propagation velocity in the coupled region, which is slower than that in the uncoupled region $\left(v=\sigma_{x y} / c_{c h}\right)$ since $\delta \geq 0$. The EMP in the coupled region is not chiral; there are modes propagating in the forward (or right) and backward (or left) directions along the $x$ axis. By expanding current density using eigenspinors of the $2 \times 2$ matrix, we have for $x \in[0, \ell]$

$$
\left(\begin{array}{c}
j_{1}(x) \\
j_{2}(x)
\end{array}\right)=\alpha_{R}\left(\begin{array}{c}
1 \\
-r
\end{array}\right) e^{+i \frac{\omega}{v_{c}} x}-\alpha_{L}\left(\begin{array}{c}
-r \\
1
\end{array}\right) e^{-i \frac{\omega}{v_{c}} x}
$$

where

$$
r \equiv \frac{1-\delta-\sqrt{1-2 \delta}}{\delta}, \quad\left(v_{c}=\frac{1-r}{1+r} v\right) .
$$

Because $\delta$ is an increasing function of $c_{x}$ with upper bound $1 / 2$, we define the weak and strong coupling limit as $\delta \rightarrow 0$ and $1 / 2$ (or $r \rightarrow 0$ and 1 ), respectively. The first term on the right-hand side of Eq. (10) represents the mode propagating with the positive velocity in the coordinate $x$ with amplitude $\alpha_{R}$. Using the continuity equation, or by substituting Eq. (10) into Eq. (8), we obtain the charge density

$$
\left(\begin{array}{l}
\rho_{1}(x) \\
\rho_{2}(x)
\end{array}\right)=\frac{1}{v_{c}}\left\{\alpha_{R}\left(\begin{array}{c}
1 \\
-r
\end{array}\right) e^{+i \frac{\omega}{v_{c}} x}+\alpha_{L}\left(\begin{array}{c}
-r \\
1
\end{array}\right) e^{-i \frac{\omega}{v_{c}} x}\right\} .
$$

Next, we examine the boundary conditions to be satisfied for the boundaries of the coupled region at $x=0$ and $\ell$. By the spatial integration of the continuity equation over an infinitesimal region including the boundary, it is shown that the current must be continuous there;

$$
\lim _{\epsilon \rightarrow 0} \int_{x^{\prime}-\epsilon}^{x^{\prime}+\epsilon} d x \partial_{x} j_{1}(x, t)=-\partial_{t} \int_{x^{\prime}-\epsilon}^{x^{\prime}+\epsilon} d x \rho_{1}(x, t) \rightarrow j_{i}\left(x^{\prime}+0\right)=j_{i}\left(x^{\prime}-0\right) .
$$

Therefore, by setting $j_{1}(0)=a, j_{1}(\ell)=\tilde{a}, j_{2}(0)=-\tilde{b}$, and $j_{2}(\ell)=-b$, we obtain from Eq. (10)

$$
\begin{aligned}
& a=\alpha_{R}+r \alpha_{L}, \quad-\tilde{b}=-r \alpha_{R}-\alpha_{L}, \\
& \tilde{a}=\alpha_{R} e^{i \frac{\omega}{v_{c}} \ell}+r \alpha_{L} e^{-i \frac{\omega}{v_{c}} \ell}, \quad-b=-r \alpha_{R} e^{i \frac{\omega}{v_{c}} \ell}-\alpha_{L} e^{-i \frac{\omega}{v_{c}} \ell} .
\end{aligned}
$$


We note that the charge density is not continuous at the boundaries. Such a discontinuity is easy to recognize by considering a square wave of width $\Delta x$ as an incident wave prepared in the uncoupled region. When it enters the coupled region, the width must decrease to $\frac{v_{c}}{v} \Delta x$ and the charge density must increase because of the charge conservation. Even though the discontinuity of the charge density, by itself, does not result in any serious error, it might represent poor modeling on the boundary. Indeed, according to Volkov's theory, $\ell_{x}$ actually depends on $\kappa$, so $\ell_{x}$ may be changed at the boundary. There is a possibility that a charge flow in the direction perpendicular to the edge may exist at the boundary. In this manuscript, we ignored the possible effect due to the discontinuous change in the charge density.

By eliminating $\alpha_{R}$ and $\alpha_{L}$ from the above equations, we get a $2 \times 2$ symplectic (transfer) matrix with a unit determinant that relates the current density of one domain to that of the other domain as

$$
\left(\begin{array}{c}
a \\
\tilde{a}
\end{array}\right)=T(\omega)\left(\begin{array}{l}
\tilde{b} \\
b
\end{array}\right)
$$

where

$$
T(\omega) \equiv\left(\begin{array}{cc}
1 & 1 \\
e^{i \frac{\omega}{v_{c}} \ell} & e^{-i \frac{\omega}{v_{c}} \ell}
\end{array}\right)\left(\begin{array}{cc}
\frac{1}{r} & 0 \\
0 & r
\end{array}\right)\left(\begin{array}{cc}
1 & 1 \\
e^{i \frac{\omega}{v_{c}} \ell} & e^{-i \frac{\omega}{v_{c}} \ell}
\end{array}\right)^{-1}=\frac{1}{-2 i r \sin \left(\frac{\omega \ell}{v_{c}}\right)}\left(\begin{array}{cc}
t_{\omega} & -t_{0} \\
t_{0} & -t_{\omega}^{*}
\end{array}\right),
$$

and $t_{\omega} \equiv e^{-i \frac{\omega}{v_{c}} \ell}-r^{2} e^{+i \frac{\omega}{v_{c}} \ell}$ (and therefore $t_{0}=1-r^{2}$ ). Because an EMP propagates freely in the uncoupled region of each domain, we have a phase relationship between $a(b)$ and $\tilde{a}$ $(\tilde{b})$ as follows:

$$
\left\{\begin{aligned}
e^{+i \frac{\omega\left(L_{1}-\ell\right)}{v}} \tilde{a} & =a \\
e^{+i \frac{\omega\left(L_{2}-\ell\right)}{v}} \tilde{b} & =b .
\end{aligned}\right.
$$

Substituting Eq. (17) into Eq. (15), we obtain

$$
\left(\begin{array}{c}
1 \\
e^{-i \frac{\omega\left(L_{1}-\ell\right)}{v}}
\end{array}\right) a=T(\omega)\left(\begin{array}{c}
e^{-i \frac{\omega\left(L_{2}-\ell\right)}{v}} \\
1
\end{array}\right) b
$$

By multiplying $\left(1,-e^{i \frac{\omega\left(L_{1}-\ell\right)}{v}}\right)$ with the both sides of Eq. (18), we obtain the equation written as

$$
\operatorname{Re}\left[e^{-i \frac{\omega}{v}\left(\frac{L_{1}+L_{2}}{2}-\ell\right)} t_{\omega}\right]=t_{0} \cos \left(\frac{\omega}{2 v}\left(L_{1}-L_{2}\right)\right)
$$


which determines the possible eigenfrequencies. This is simplified when the two domains are geometrically equivalent, i.e., $L_{1}=L_{2} \equiv L$, as

$$
r^{2} \sin ^{2}\left(\frac{\omega(L-\ell)}{2 v}-\frac{\omega \ell}{2 v_{c}}\right)=\sin ^{2}\left(\frac{\omega(L-\ell)}{2 v}+\frac{\omega \ell}{2 v_{c}}\right) .
$$

This equation can be solved numerically in general and analytically in a certain limit.

Figure 2(a) shows the numerical solution of Eq. (20) for $L=6 \ell$ as a function of the coupling strength $(r)$. The interaction always decreases the frequency. In the weak coupling limit, there are two fundamental modes with equal angular frequency $\omega=2 \pi v / L$. The energies of the originally degenerate states are split and cross again (at $r \simeq 0.66$ ) by increasing capacitive coupling. The possible crossing points and behavior in a strong coupling regime can be understood on physical grounds, by introducing the following two modes. One physically expected mode has the fundamental frequency

$$
\omega_{e}=\frac{\pi v}{L-\ell}
$$

which corresponds to a new EMP mode moving around the periphery of the two coupled domains [see Fig. 2(b)]. The eigenfrequency of the other mode is a multiple of

$$
\omega_{s}=\frac{\pi v_{c}}{\ell}
$$

which represents a standing wave localized in the coupled region [see Fig. 2(c)]. This becomes the lowest frequency mode in the strong coupling limit, while it is a high-frequency mode in weak coupling. Please note that the $T(\omega)$ matrix is ill-defined exactly when $\omega=n \omega_{s}$ and that Eq. (14) gives $\tilde{a}=a(-1)^{n}$ and $\tilde{b}=b(-1)^{n}$, which are inconsistent with the phase condition of Eq. (17). Thus, even for a strong coupling case, the calculated frequencies in Fig. 2(a) are very slightly displaced from $n \omega_{s}$.

The level crossing between $\omega_{s}$ and $\omega_{e}$ occurs when $r=1-\frac{2 \ell}{L}\left(\equiv r_{c}\right)$. The critical coupling strength is determined by the geometrical parameters $\ell$ and $L$ only. The spectrum at the critical point exhibits a special feature that the possible frequencies are exact multiples of the fundamental frequency.

By multiplying $\left(1, e^{i \frac{\omega(L-\ell)}{v}}\right)$ with the both sides of Eq. (18), we know that

$$
\frac{a}{b}=\frac{1+r^{2}}{2 r} \cos \left(\frac{\omega(L-\ell)}{v}\right)+\frac{1-r^{2}}{2 r} \sin \left(\frac{\omega(L-\ell)}{v}\right) \cot \left(\frac{\omega \ell}{v_{c}}\right)
$$


(a)

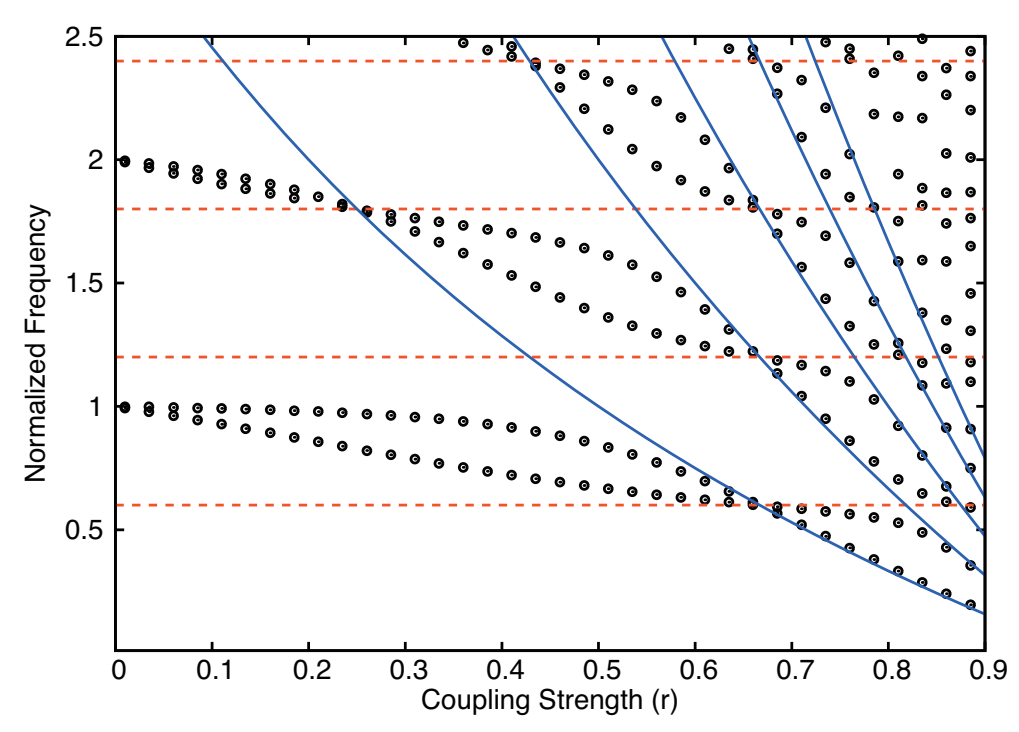

(b)

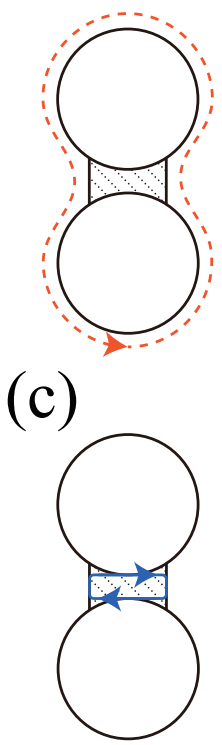

FIG. 2. Low-energy spectrum of a simplest EMP molecule. (a) The calculated frequency is plotted as dots in units of the fundamental frequency of an isolated domain without the interaction $\left(\frac{2 \pi v}{L}\right)$. This result is for $L=6 \ell$. The dashed lines represent new EMP modes $\left(\omega_{e}, 2 \omega_{e}, 3 \omega_{e}\right.$ and $\left.4 \omega_{e}\right)$ that propagate along the outer edge (b), and the solid curves represent the localized standing wave modes $\left(\omega_{s}, 2 \omega_{s}, 3 \omega_{s}, 4 \omega_{s}\right.$, and $\left.5 \omega_{s}\right)$ in the coupled region (c).

holds for the general value of $\omega$. Meanwhile, solutions of Eq. (20) satisfy either

$$
-r \sin \left(\frac{\omega(L-\ell)}{2 v}-\frac{\omega \ell}{2 v_{c}}\right)=\sin \left(\frac{\omega(L-\ell)}{2 v}+\frac{\omega \ell}{2 v_{c}}\right)
$$

or

$$
r \sin \left(\frac{\omega(L-\ell)}{2 v}-\frac{\omega \ell}{2 v_{c}}\right)=\sin \left(\frac{\omega(L-\ell)}{2 v}+\frac{\omega \ell}{2 v_{c}}\right) .
$$

It is shown by combining Eqs. (23) and (24) or (25) that $a / b$ must be +1 or -1 in order that the solutions exist for the general coupling strength. In the weak coupling limit, the higher (lower) frequency state has $a / b=+1(-1)$. The higher or lower frequency characteristics change when the two modes cross each other with increasing $r$.

Putting $a / b=+1(-1)$ into Eq. (14), we obtain $\alpha_{R}=e^{-i \frac{\omega}{v_{c}} \ell} \alpha_{L}\left(\alpha_{R}=-e^{-i \frac{\omega}{v_{c}} \ell} \alpha_{L}\right)$, by which Eqs. (10) and (12) are determined with the exception of the normalization factor. 
The current and charge densities for $a / b=+1$ are

$$
\begin{aligned}
& \left(\begin{array}{l}
j_{1}(x) \\
j_{2}(x)
\end{array}\right)_{+1}=\alpha_{L} e^{-i \frac{\omega \ell}{2 v_{c}}}\left(\begin{array}{c}
(1+r) \cos \left(\frac{\omega}{v_{c}}\left(x-\frac{\ell}{2}\right)\right)+i(1-r) \sin \left(\frac{\omega}{v_{c}}\left(x-\frac{\ell}{2}\right)\right) \\
-(1+r) \cos \left(\frac{\omega}{v_{c}}\left(x-\frac{\ell}{2}\right)\right)+i(1-r) \sin \left(\frac{\omega}{v_{c}}\left(x-\frac{\ell}{2}\right)\right)
\end{array}\right), \\
& \left(\begin{array}{l}
\rho_{1}(x) \\
\rho_{2}(x)
\end{array}\right)_{+1}=\frac{\alpha_{L} e^{-i \frac{\omega \ell}{2 v_{c}}}}{v_{c}}\left(\begin{array}{l}
(1-r) \cos \left(\frac{\omega}{v_{c}}\left(x-\frac{\ell}{2}\right)\right)+i(1+r) \sin \left(\frac{\omega}{v_{c}}\left(x-\frac{\ell}{2}\right)\right) \\
(1-r) \cos \left(\frac{\omega}{v_{c}}\left(x-\frac{\ell}{2}\right)\right)-i(1+r) \sin \left(\frac{\omega}{v_{c}}\left(x-\frac{\ell}{2}\right)\right)
\end{array}\right) .
\end{aligned}
$$

The current and charge densities for $a / b=-1$ are given by exchanging current with charge for $a / b=+1$ as

$$
\left(\begin{array}{l}
j_{1}(x) \\
j_{2}(x)
\end{array}\right)_{-1}=-v_{c}\left(\begin{array}{l}
\rho_{1}(x) \\
\rho_{2}(x)
\end{array}\right)_{+1},\left(\begin{array}{l}
\rho_{1}(x) \\
\rho_{2}(x)
\end{array}\right)_{-1}=-\frac{1}{v_{c}}\left(\begin{array}{l}
j_{1}(x) \\
j_{2}(x)
\end{array}\right)_{+1} .
$$

Since sine terms vanish at the center of the coupled region $(x=\ell / 2)$ for any $\omega$, we first assume the convention that the normalization factor of $\alpha_{L} e^{-i \frac{\omega \ell}{2 v_{c}}}$ is a real number. Then the signs of $a / b$ represent different configurations of the dipole moments. For $a / b=+1$, the charge densities at the two domains in the coupled region have the same sign (like "antibonding orbital"). The direction of the dipole moment in each domain points in the opposite direction, and a net dipole moment of the two domains vanishes in total. Meanwhile, for $a / b=-1$, the charge densities at the two domains in the coupled region have different signs (like "bonding orbital"). The direction of the dipole moment of each domain points in the same direction, and the two domains constructively make a large dipole moment as a whole. The above convention is invalid unless $\alpha_{L} e^{-i \frac{\omega \ell}{2 v_{c}}}$ is a real number, because the normalization factor is a complex number in general. For example, in a strong coupling region, $\omega=n \omega_{s}$ and the normalization factor of $\alpha_{L}(-i)^{n}$, where $\alpha_{L}$ is a real number. The dipole moment characteristics change as $r$ increases. Generally, we can specify the phase of $\alpha_{L}$ for a given $\omega$, because $\left[j_{1}(0)\right]_{ \pm 1}=\left( \pm r+e^{-i \frac{\omega \ell}{v_{c}}}\right) \alpha_{L}$ is a real number.

We consider a geometrical case of $L \gg \ell$ in which an incident steady current flows in the first domain towards the coupled region. This situation is expressed by setting $a=1$ in Eq. (15). Because the EMP of the second domain propagates in the counterclockwise direction as shown in Fig. 1(b), it takes a very long time to arrive at $x=\ell$ from $x=0$. We therefore may assume that $b=0$ in Eq. (15). From these conditions, we obtain the reflectance and transmittance as

$$
R \equiv|\tilde{a}|^{2}=\left|\frac{\left(1-r^{2}\right) e^{i \frac{\omega \ell}{v_{c}}}}{1-r^{2} e^{2 i \frac{\omega \ell}{v_{c}}}}\right|^{2}, \quad T \equiv|\tilde{b}|^{2}=\left|\frac{r\left(1-e^{2 i \frac{\omega \ell}{v_{c}}}\right)}{1-r^{2} e^{2 i \frac{\omega \ell}{v_{c}}}}\right|^{2} .
$$


This result coincides with the result known for the reflection and transmission of light by thin films $\stackrel{23,24}{2}$ The coupled region can be expressed as a non-absorbing medium with the refractive index of $n \equiv v / v_{c}$ or $n=(1+r) /(1-r)$. When the frequency of an incident wave matches the frequency of a standing wave (i.e. when $\omega$ is a multiple of $\omega_{s}$ ), perfect reflection with $R=1$ and $T=0$ is realized. In the strong coupling limit, nearly perfect transmission is expected when $\omega=\left(n+\frac{1}{2}\right) \omega_{s}$, where $n=0,1, \cdots$.

\section{PERIODIC DOMAINS}

In this section, we apply the formulation presented for the simplest EMP molecule in the preceding sections to periodic structures of planar EMP crystals, including a chain, ladder, and honeycomb network composed of $N$ domains. To simplify the analysis, we introduce the following vector notation for the two-component column matrix:

$$
\mathbf{a}_{i} \equiv\left(\begin{array}{c}
\tilde{a}_{i} \\
a_{i}
\end{array}\right), \quad \tilde{\mathbf{a}}_{i} \equiv\left(\begin{array}{c}
a_{i} \\
\tilde{a}_{i}
\end{array}\right) .
$$

Note that a tilde rule is adopted, namely the amplitude with a tilde is located in the first (second) component of $\mathbf{a}_{i}\left(\tilde{\mathbf{a}}_{i}\right)$.

\section{A. Chain}

A straight chain is formed when $N$ domains are aligned along a line. Figure 3(a) shows the constituents of the chain, where the amplitudes of the vertices are related to each other by the boundary condition of the coupled region as $\tilde{\mathbf{a}}=T(\omega) \mathbf{b}$ and by a phase relationship of the uncoupled region as $\mathbf{c}=R_{\frac{L}{2}-\ell}(\omega) \tilde{\mathbf{a}}$. Here, $R_{\frac{L}{2}-\ell}(\omega)$ originates from the phase accumulation caused by free propagation of EMPs with a fixed chirality from $\tilde{c}$ to $a$ and from $\tilde{a}$ to $c$ whose circular distance is $\frac{L}{2}-\ell$ :

$$
R_{\frac{L}{2}-\ell}(\omega)=\left(\begin{array}{cc}
e^{-i \frac{\omega}{v}\left(\frac{L}{2}-\ell\right)} & 0 \\
0 & e^{+i \frac{\omega}{v}\left(\frac{L}{2}-\ell\right)}
\end{array}\right) .
$$

The elimination of ã gives $\mathbf{c}=R_{\frac{L}{2}-\ell}(\omega) T(\omega) \mathbf{b}$. Because $\operatorname{det}\left(R_{\frac{L}{2}-\ell}(\omega) T(\omega)\right)=1$ holds for any $\omega$, we know from Bloch's theorem the existence of a unitary matrix $U$ and phase $\theta$ 
(a)

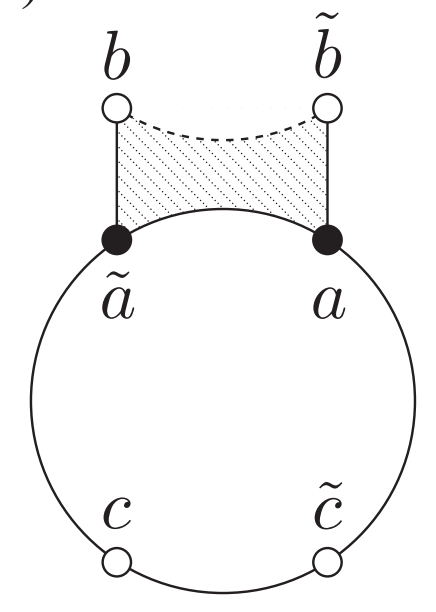

(b)

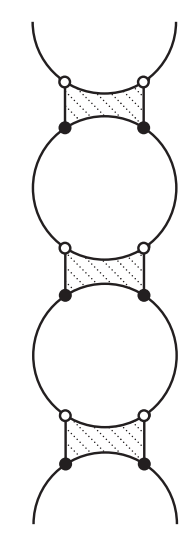

(c)

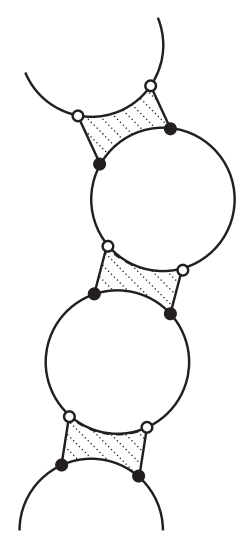

FIG. 3. Geometries of chain. (a) The basic unit of a chain consists of a domain and coupled region. Note that the domain shape is arbitrary and that we assume it to be a circle here. The circumference of a domain is $L$, and the length of a coupled region is $\ell$. (b) When the circumferential distance on a domain between the two vertices $\tilde{c}$ and $a$ is equal to that between $\tilde{a}$ and $c(\overline{\tilde{c} a}=\overline{\tilde{a} c}=$ $\left.\frac{L}{2}-\ell\right)$, the chain is straight. Otherwise $\left(\overline{\tilde{c} a}=\frac{L}{2}-\ell-R_{j}\right.$ and $\overline{\tilde{a} c}=\frac{L}{2}-\ell+R_{j}$ for a $j$ th domain $)$, the chain is deformed, as shown in (c).

$(\in[0, \pi])$ that satisfy

$$
U R_{\frac{L}{2}-\ell}(\omega) T(\omega) U^{\dagger}=\left(\begin{array}{cc}
e^{-i \theta} & 0 \\
0 & e^{+i \theta}
\end{array}\right)
$$

This is consistent with the characteristic equation $\lambda^{2}-\operatorname{tr}\left(R_{\frac{L}{2}-\ell}(\omega) T(\omega)\right) \lambda+1=0$, and one may assume that the eigenvalues of $R_{\frac{L}{2}-\ell}(\omega) T(\omega)$ are $e^{ \pm i \theta}$. Equation (32) leads to the relation $\operatorname{tr}\left(R_{\frac{L}{2}-\ell}(\omega) T(\omega)\right)=2 \cos \theta$, which is

$$
\cos \theta=\frac{1+r^{2}}{2 r} \cos \left(\frac{\omega}{v}\left(\frac{L}{2}-\ell\right)\right)+\frac{1-r^{2}}{2 r} \sin \left(\frac{\omega}{v}\left(\frac{L}{2}-\ell\right)\right) \cot \left(\frac{\omega \ell}{v_{c}}\right) .
$$

$\theta$ may be determined from Eq. (33) as a function of $\omega$, which also specifies the dispersion relation of a chain. Because the periodicity of $N$ domains is characterized by the boundary condition $[R(\omega) T(\omega)]^{N}=I$, this condition discretizes $\theta$ through a constraint $N \theta=2 \pi n$, where $n$ is the wavenumber, and $\theta$ may be regarded as a continuum when $N \rightarrow \infty$.

The band structure depends strongly on the coupling strength, as shown in Fig. 4(a) for coupling constants $r=0.2$ [left], 0.4 [middle] and 0.8[right]. For a weak coupling $(r=0.2)$, a 
weak dispersive band appears near the fundamental excitation mode of a domain $\left(\omega=\frac{2 \pi v}{L}\right)$, and energy gaps are formed between the subbands. When $r=0.4$, the dispersive nature (or the bandwidth) is almost doubled. There is a strong similarity between the band structures shown in Fig. 4(a) and miniband structures calculated from the Kronig-Penny model for periodic semiconductor superlattices. ${ }^{25-27}$ Indeed, as we will show in Appendix B, the $T(\omega)$ matrix in Eq. (32) can be constructed from physical variables of a binary superlattice.

In a strong coupling $(r=0.8)$, the bandwidth of each subband is suppressed. An overlap between the calculated dispersion and a linear dispersion of $\omega=\frac{v}{L / 2-\ell} \theta$ [as expressed by red dashed lines in Fig. [4(a)] can be found at intervals. Since $L / 2-\ell$ is the effective unit-cell length along a chain, the linear dispersion may be expressed as $\omega=v k$, where $k$ is the wavevector along the chain and Eq. (32) shows that $\omega=v k$ becomes exact in the strongcoupling limit because $T(\omega)$ becomes a unit matrix. In fact, because the right-hand side of Eq. (33) is singular at a multiple of $\omega_{s}$, the subbands are separated by energy gaps formed at around $n \omega_{s}$. In the gaps, $\theta$ is an imaginary number giving localized states. The linear dispersion continuously changes into a flat band that represents the standing waves. A flat band is mostly composed of the localized standing waves and is associated with a small component of a chiral wave in the uncoupled regions. These dispersionless modes do not propagate along the chain. A linear dispersion is mostly composed of the chiral wave in the uncoupled regions and is associated with a small component of the standing waves. These dispersive modes propagate along the chain. The panels in Fig. 4(b) and (c) show these eigenmodes.

Since an ideal chain with a perfect periodicity does not exist in nature, we shall discuss a geometrical deformation of a chain. When a straight chain is geometrically deformed locally by $R_{j}$, as shown in Fig. $4(\mathrm{c})$, the matrix $R(\omega)$ acquires a $U(1)$ phase. The periodic boundary condition is modified as

$$
\prod_{j=1}^{N}\left[e^{+i \frac{\omega}{v} R_{j}}\left(\begin{array}{cc}
e^{-i \frac{\omega}{v}\left(\frac{L}{2}-\ell\right)} & 0 \\
0 & e^{+i \frac{\omega}{v}\left(\frac{L}{2}-\ell\right)}
\end{array}\right) T(\omega)\right]=I .
$$

When $R \equiv \sum_{j=1}^{N} R_{j}=0$, the effect of the local deformation is removed, which is similar to the pure gauge degree of freedom in gauge theories. When $R \neq 0$, a chain is not a straight line but a closed curve. Such a change in global topology does not alter the band structure 

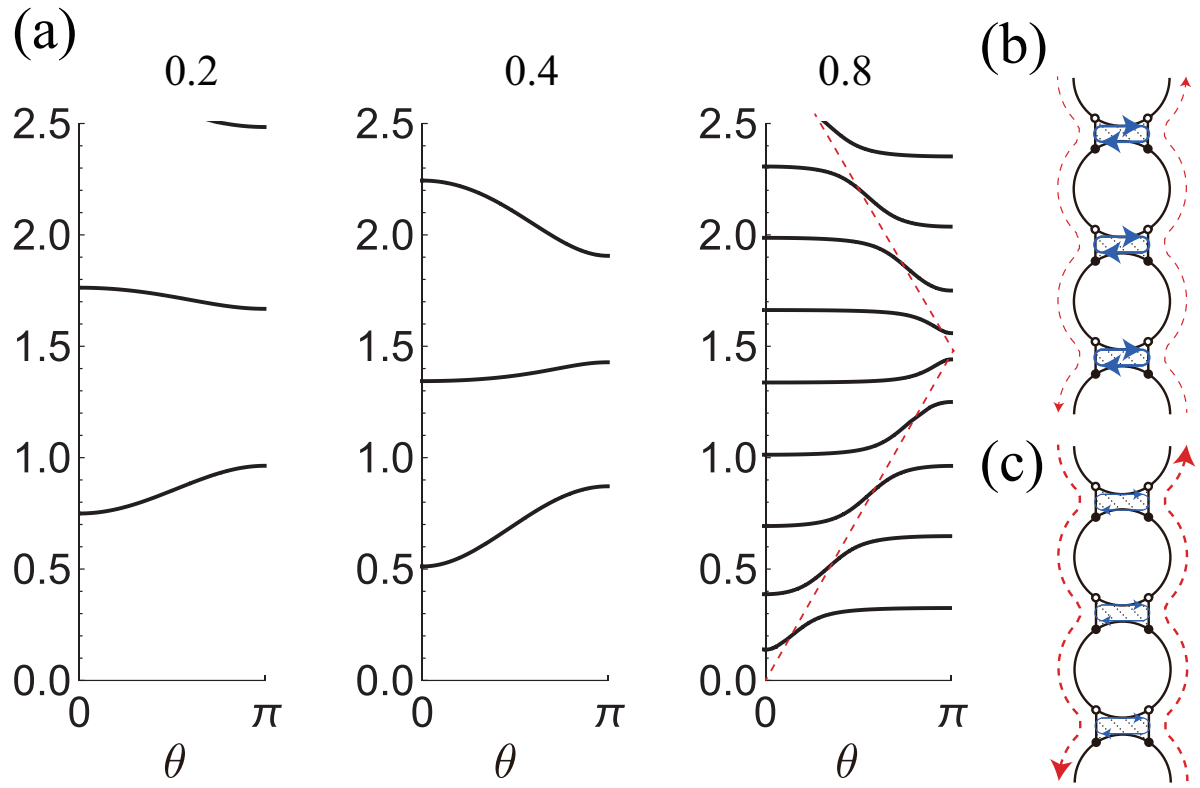

FIG. 4. Band structures of a chain. (a) The dispersion is given as a function of $\theta \in[0, \pi]$ for different coupling strength $r=0.2,0.4$, and 0.8. $\omega$ is normalized in units of $\frac{2 \pi v}{L}$ with $L=6 \ell$. Red dashed lines are the chiral dispersion of the original EMP of an isolated domain, which is the eigenmode at the strong coupling limit. We note that There is an energy gap at $\theta=0$ which vanishes at the strong coupling limit as $\frac{\omega}{\frac{2 \pi v}{L}}=\frac{1}{\pi} \sqrt{\frac{(1-r)^{2}}{r r_{c}^{2}\left(1-r_{c}\right)}}$. (b) and (c) illustrate the eigenmodes of a flat band and linear dispersion, respectively.

but may cause a physical effect, namely a shift in the wavenumber

$$
\theta=\frac{2 \pi}{N}\left(n-\frac{\omega R}{2 \pi v}\right)
$$

We apply this result to understand the effect of a geometrical change from a straight line to a square. Suppose eight domains $(N=8)$ with $L=8 \ell$ are aligned to form a straight line. It can be deformed into a square by setting $R=8 \ell$. In the weak coupling limit, we may assume $\omega=\frac{2 \pi v}{L}$. By putting it into Eq. (35), we have $\theta=\frac{2 \pi}{N}(n-1)$. However, a shift in $\theta$ would be difficult to validate experimentally because a planar periodic crystal must be modified to obtain an output signal. A $U(1)$ phase can be irrelevant to physical observables like the reflectance or transmittance, because they are given by the amplitude absolute square.

The results for the two coupled domains, such as in Fig. 2(a) in Sec. III, are approximately embedded into the band structure in Fig. 因(a) at $\theta=0$ and $\pi$. Specifically, for the weak coupling, we may rewrite Eq. (23) as $a / b=-\cos \theta$ by using Eq. (33). This is shown by 
replacing $L$ in Eq. (23) with $\frac{L}{2}$, and the remaining $\frac{L}{2}$ is used to obtain the minus sign in $a / b=-\cos \theta$, where the phase relationship between $a$ and $b$ is reversed for the case that $\omega \simeq \frac{2 \pi v}{L}$ because $\tan (x+\pi)=\tan (x)$ and $\cos (x+\pi)=-\cos (x)$.

\section{B. Ladder}

We obtain a straight ladder by interconnecting the two basic units of a chain as shown in Fig. 5(a) and by identifying $\mathbf{c}_{2}$ and $\mathbf{c}_{1}$ with $\mathbf{a}_{3}$ and $\mathbf{b}_{1}$, respectively, as shown in Fig. 5(b). Due to the chirality, a ladder includes two input channels (say $\tilde{c}_{1}$ and $\tilde{c}_{2}$ ). Thus, a ladder corresponds to a device that can reflect or transmit the two wave signals.

(a)

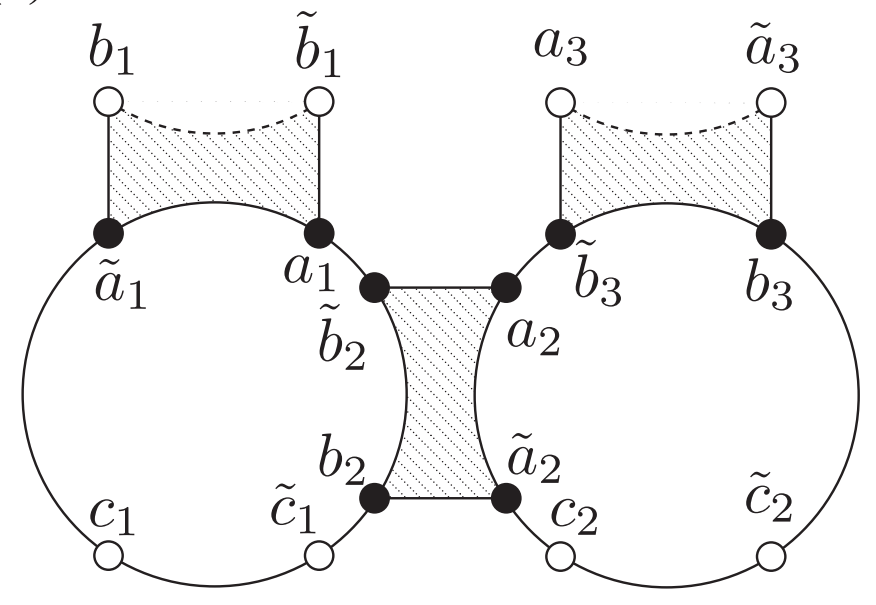

(b)

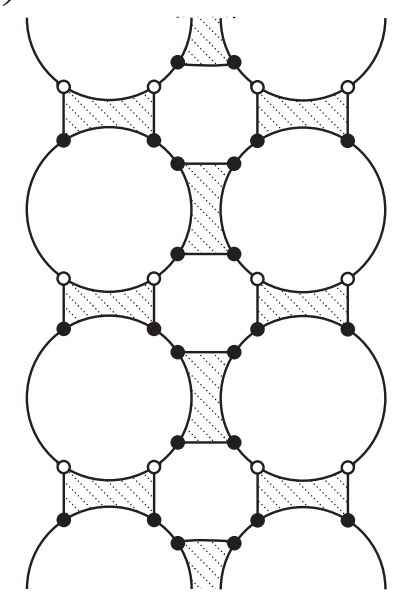

FIG. 5. Geometry of ladder. (a) The basic unit of a ladder consists of the two domains and three coupled regions. The circumference of a domain is $L$, and the length of a coupled region is $\ell$. The circumferential distance between $a_{1}$ and $\tilde{b}_{2}\left(\overline{a_{1} \tilde{b}_{2}}\right)$ is equal to $\overline{a_{2} \tilde{b}_{3}}, \overline{b_{2} \tilde{c}_{1}}$, and $\overline{\tilde{a}_{2} c_{2}}$, which is given by $s \equiv \frac{L}{4}-\ell$. (b) The domain structure of a straight ladder.

To calculate its band structure, we need to construct a $4 \times 4$ matrix that satisfies

$$
\left(\begin{array}{l}
\mathbf{c}_{2} \\
\mathbf{c}_{1}
\end{array}\right)=M(\omega)\left(\begin{array}{l}
\mathbf{a}_{3} \\
\mathbf{b}_{1}
\end{array}\right) .
$$

In addition to the $T$ matrix satisfying $\tilde{\mathbf{b}}_{3}=T(\omega) \mathbf{a}_{3}$ and $\tilde{\mathbf{a}}_{1}=T(\omega) \mathbf{b}_{1}$, let us introduce a 
$2 \times 2$ matrix for the interconnected region between the two domains.

$$
\left(\begin{array}{l}
\tilde{a}_{2} \\
b_{2}
\end{array}\right)=S(\omega)\left(\begin{array}{l}
a_{2} \\
\tilde{b}_{2}
\end{array}\right) .
$$

This $S(\omega)$ matrix is known from the boundary condition Eq. (14) as

$$
S(\omega) \equiv U R_{c}(\omega) U^{-1}=\cos \left(\frac{\omega \ell}{v_{c}}\right) I+i \sin \left(\frac{\omega \ell}{v_{c}}\right) W
$$

where

$$
U \equiv\left(\begin{array}{ll}
1 & r \\
r & 1
\end{array}\right), \quad R_{c}(\omega) \equiv\left(\begin{array}{cc}
e^{+i \frac{\omega \ell}{v_{c}}} & 0 \\
0 & e^{-i \frac{\omega \ell}{v_{c}}}
\end{array}\right), \quad W=\frac{1+r^{2}}{1-r^{2}}\left(\begin{array}{cc}
1 & -\frac{2 r}{1+r^{2}} \\
\frac{2 r}{1+r^{2}} & -1
\end{array}\right)
$$

We note that because $W^{2}=I, S(\omega)=e^{i\left(\frac{\omega \ell}{v_{c}}\right) W}$. This expression may be used to simplify some calculation.

For the free propagation of the EMP in the uncoupled regions, we have

$$
\left(\begin{array}{c}
a_{2} \\
\tilde{b}_{2}
\end{array}\right)=R_{s}(\omega)\left(\begin{array}{c}
\tilde{b}_{3} \\
a_{1}
\end{array}\right), \quad R_{s}(\omega) \equiv\left(\begin{array}{cc}
e^{+i \frac{\omega s}{v}} & 0 \\
0 & e^{-i \frac{\omega s}{v}}
\end{array}\right)
$$

Therefore, we obtain

$$
\begin{aligned}
& \left(\begin{array}{c}
c_{2} \\
\tilde{c}_{1}
\end{array}\right)=R_{s}(\omega) S(\omega) R_{s}(\omega)\left(\begin{array}{c}
\tilde{b}_{3} \\
a_{1}
\end{array}\right), \\
& \left(\begin{array}{c}
\tilde{c}_{2} \\
c_{1}
\end{array}\right)=\left(\begin{array}{cc}
e^{-i \frac{\omega}{v}\left(\frac{L}{2}-\ell\right)} & 0 \\
0 & e^{+i \frac{\omega}{v}\left(\frac{L}{2}-\ell\right)}
\end{array}\right)\left(\begin{array}{l}
b_{3} \\
\tilde{a}_{1}
\end{array}\right) .
\end{aligned}
$$

Finally, the explicit form of the $4 \times 4$ matrix $M(\omega)$ is given by

$$
M(\omega)=\left(\begin{array}{ccc}
e^{-i \frac{\omega}{v}\left(\frac{L}{2}-\ell\right)} & 0 & 0 \\
0 & R_{s}(\omega) S(\omega) R_{s}(\omega) & 0 \\
0 & 0 & e^{+i \frac{\omega}{v}\left(\frac{L}{2}-\ell\right)}
\end{array}\right)\left(\begin{array}{cc}
T(\omega) & 0 \\
0 & T(\omega)
\end{array}\right) .
$$

The characteristic equation of $M(\omega)$ is written as a symmetric form $\lambda^{4}+A(\omega) \lambda^{3}+B(\omega) \lambda^{2}+$ $A(\omega) \lambda+1=0$, with functions $A(\omega)=-\operatorname{tr}(M(\omega))$ and $B(\omega)=\frac{1}{2}\left(A(\omega)^{2}-\operatorname{tr}\left(M(\omega)^{2}\right)\right)$. By setting $\lambda=e^{i \theta}$, we rewrite this as

$$
\left(\cos \theta+\frac{A(\omega)}{4}\right)^{2}=\frac{8+A(\omega)^{2}-4 B(\omega)}{16} .
$$


By solving it with respect to $\theta$, we plot the dispersion relation in Fig. 6(a) for coupling constants $(r=0.2$ [left], 0.4 [middle], and 0.75 [right]). For a weak coupling $(r=0.2)$, the dispersion appears near $\omega=\frac{2 \pi v}{L}$, which is the fundamental excitation mode of a domain. According to the two domains in the unit cell of a ladder, two dispersion curves appear as a pair in the weak coupling. For a strong coupling $(r=0.75)$, the standing wave modes $\left(\omega_{s}\right)$ appear as flat bands between 0.5 and 0.6. These modes are also localizing at an interconnected region between the two domains of a unit cell. They are nearly degenerate because $S\left(n \omega_{s}\right)=(-1)^{n} I$ holds and therefore Eq. (43) consists of the same $2 \times 2$ matrix in a diagonal form.

In the strong coupling limit, since $T(\omega) \rightarrow 1$, we can expect that the possible modes of the system are divided into a counter propagating (outer) edge modes and other inner modes. The latter -EMPs rotating around each hole of the system- have a higher energy $\omega=\frac{\pi v}{2 s}$, which is visible as an almost flat band.

(a)

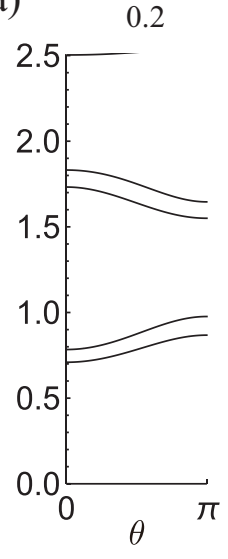

0.4

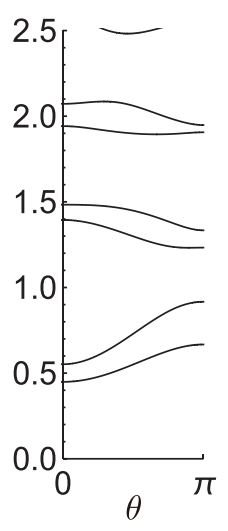

0.75

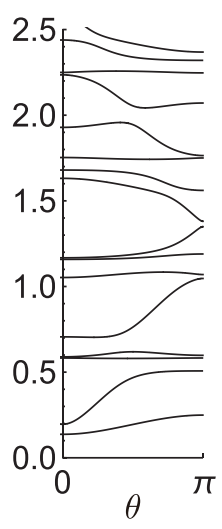

(b)

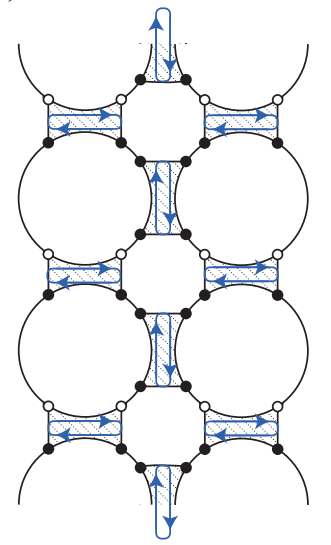

(c)

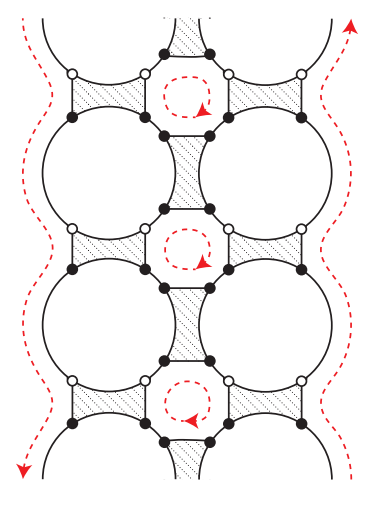

FIG. 6. Band structures of a ladder. (a) Dispersion is plotted as a function of $\theta \in[0, \pi] . \omega$ is in units of $\frac{2 \pi v}{L}$, where $L=8 \ell$ is assumed. (b) and (c) Eigenmodes of a flat band and linear dispersion, respectively.

\section{Honeycomb}

As shown in Figs. 7(a) and (b), a honeycomb network can be obtained by slightly modifying the basic unit of a ladder. The circumferential distance between all nearest neighbor 
vertices in the uncoupled regions must be the same; the circumferential distance $\overline{a_{1} \tilde{b}_{2}}$ is equal to $\overline{c_{1} \tilde{a}_{1}}, \overline{b_{2} \tilde{c}_{1}}, \overline{a_{2} \tilde{b}_{3}}, \overline{b_{3} \tilde{c}_{2}}$, and $\overline{c_{2} \tilde{a}_{2}}$.

(a)

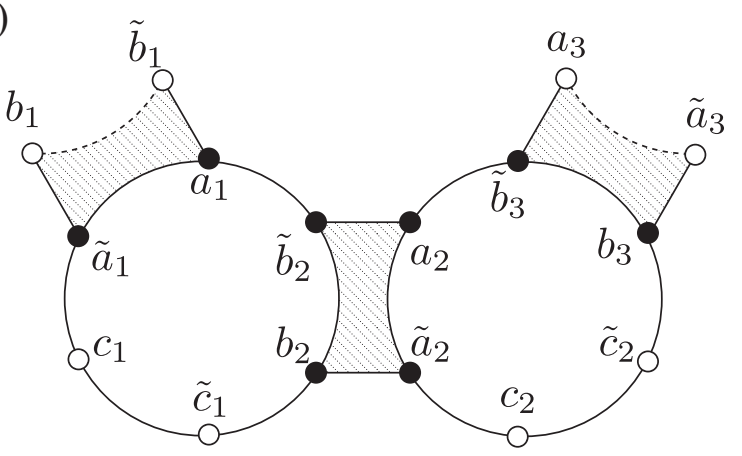

(b)

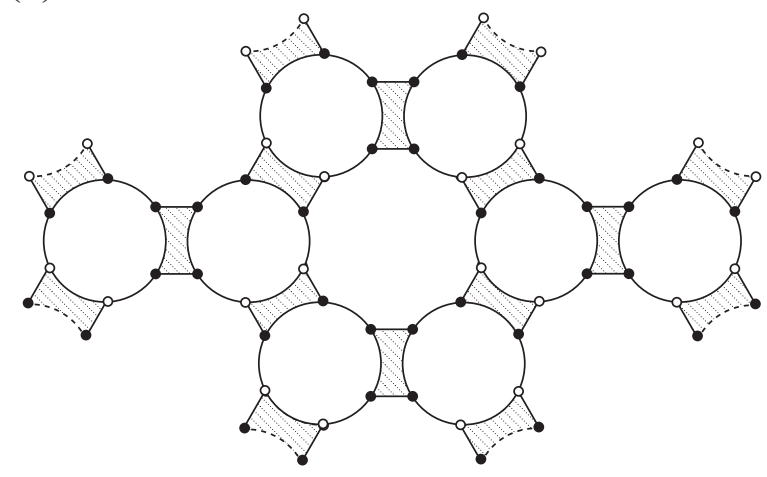

FIG. 7. Geometry of honeycomb lattice. (a) and (b) The basic unit of a honeycomb network is given by changing the vertex positions of the basic unit of a ladder.

The corresponding matrix is given by replacing $\frac{L}{2}-\ell$ of $e^{ \pm i \frac{\omega}{v}\left(\frac{L}{2}-\ell\right)}$ in Eq. (43) with $s$, where $s=\frac{L}{3}-\ell$, as

$$
M(\omega)=\left(\begin{array}{ccc}
e^{-i \frac{\omega s}{v}} & 0 & 0 \\
0 & R_{s}(\omega) S(\omega) R_{s}(\omega) & 0 \\
0 & 0 & e^{+i \frac{\omega s}{v}}
\end{array}\right)\left(\begin{array}{cc}
T(\omega) & 0 \\
0 & T(\omega)
\end{array}\right)
$$

Two adjacent units can be connected by a twisted boundary condition:

$$
\left(\begin{array}{l}
\mathbf{c}_{2} \\
\mathbf{c}_{1}
\end{array}\right)=\left(\begin{array}{cc}
0 & e^{i \theta_{1}} I \\
e^{i \theta_{2}} I & 0
\end{array}\right)\left(\begin{array}{l}
\mathbf{a}_{3} \\
\mathbf{b}_{1}
\end{array}\right)
$$

where $\theta_{1} \equiv \theta+\phi$ and $\theta_{2} \equiv \theta-\phi$. Therefore, we need to diagonalize the following $4 \times 4$ matrix.

$$
M_{h}(\omega ; \phi)=\left(\begin{array}{cc}
0 & e^{+i \phi} \\
e^{-i \phi} & 0
\end{array}\right) M(\omega) .
$$

The characteristic equation of $M_{h}$ is written as $\lambda^{4}+2 \lambda^{3} \cos \phi+\tilde{B}(\omega ; \phi) \lambda^{2}+2 \lambda \cos \phi+1=0$ where $\tilde{B}(\omega ; \phi)=2 \cos ^{2}(\phi)-\frac{1}{2} \operatorname{tr}\left(M_{h}(\omega ; \phi)^{2}\right)$. Setting $\lambda=e^{i \theta}$ leads to

$$
\left(\cos \theta+\frac{\cos \phi}{2}\right)^{2}=\frac{\cos ^{2} \phi+2-\tilde{B}(\omega ; \phi)}{4} .
$$


(a)

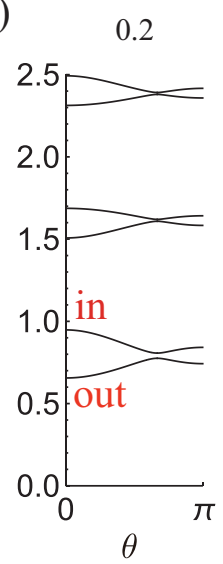

0.4

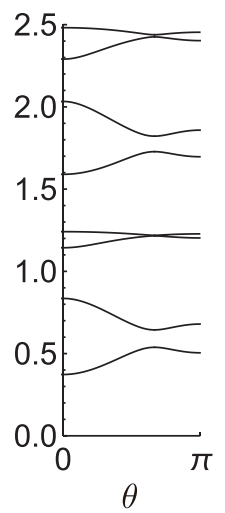

0.75

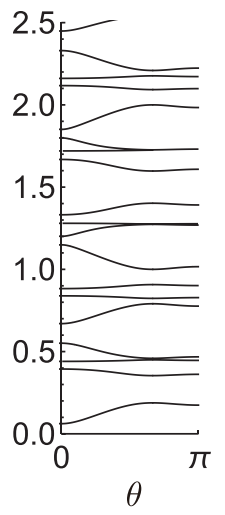

(b)

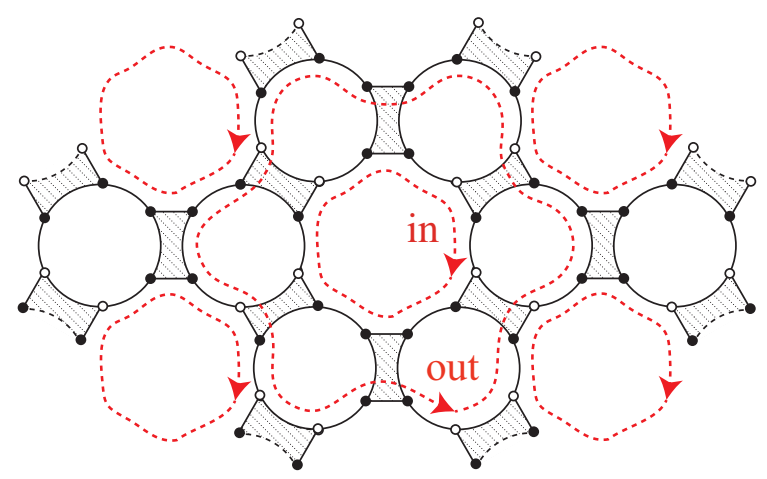

FIG. 8. Band structure of a honeycomb lattice. (a) Dispersion (along $\Gamma K$ ) is shown as a function of $\theta \in[0, \pi]$. $\omega$ is in units of $\frac{2 \pi v}{L}$, where $L=6 \ell$. (b) Two approximate eigenmodes of $\theta=0$ are illustrated by the dashed arrows.

By solving it with respect to $\theta$ with $\phi=0$, we can obtain the dispersion relation along $\Gamma K$.

Figure 8 (a) shows the band structures for coupling constants $(r=0.2$ [left], 0.4 [middle], and 0.75 [right]). For the weak coupling $(r=0.2)$, the energy band has a small energy gap at the $\mathrm{K}$ point $\left(\theta=\frac{2 \pi}{3}\right)$ for the lowest two energy subbands. It is difficult to see due to the resolution, but a small gap opens for the higher subbands. The gap of the fundamental subbands increases with increasing coupling strength.

We discuss the result using two possible modes of the system. One is the mode rotating around each hexagonal hole (or the inner edge of a hexagonal ring) [see Fig. 8(b)]. This mode has energy similar to that of the fundamental mode $\omega=\frac{2 \pi v}{L}$ and appears as the second subband at $\theta=0$ in the weak coupling regime. The other is the mode rotating around the outer edge of a hexagonal ring, which has a larger perimeter than the mode rotating around a hexagonal hole. This appears as the lowest energy subband at $\theta=0$ in the weak coupling regime. These two modes are coupled together to form real eigenmodes. In the strong coupling, the flat-band nature of the subband with the second lowest energy is noticed. The standing waves of the coupled regions are weakly interacting with each other and form a nearly flat band.

The energy band structure of honeycomb networks is not as well understood as it is for the chain. For example, the energy positions of the Dirac cone are not identified as a function of 
$\omega_{s}$. The clarification of such a problem requires more study. Note also that our honeycomb network differs greatly from a chain and ladder in the sense that it does not have an outer boundary. It is not evident for the general coupling strength whether a finite honeycomb network can support EMPs at the periphery. Introducing an outer edge to the honeycomb network would require some additional effort, which is beyond the scope of this paper. The matrix formulation we have developed for the EMP molecule and crystals is amenable to a transfer matrix method, with which we can calculate physical observables of finite periodic systems composed of $N$ domains, which we will show in detail in a subsequent paper.

\section{DISCUSSION}

Strong coupling is intriguing from various points of view, including a perfect transmission mode and flat band. Graphene has the advantage of realizing a strong coupling. Brasseur et al. achieved $r$ as large as 0.55 for two domains separated by a narrow etched line $(0.3 \mu \mathrm{m}$ width) in graphene. $\frac{28}{\underline{\underline{2}}}$ This should be compared with $r \sim 0.04$ obtained for the edge channels defined by a metal gate $(1 \mu \mathrm{m}$ width $)$ in a GaAs/AlGaAs heterostructure. $\underline{\underline{21}}^{\mathrm{w}}$ The $r$ values differ partly because the inter-edge capacitive coupling is suppressed by the screening effect of the metal gate and because the sharp edge potential of graphene prevents formation of the depletion layer (which increases virtually the width).

When two domains are positioned very close to each other for strong coupling, the validity of the description on the coupled region using a large coupling strength is not evident. Suppose that two domains merge into a single domain. The coupled region becomes the bulk region, where an EMP does not exist. The absence of low-energy excitation in the bulk is in sharp contrast to the result that many states are condensed into zero-energy in the strong coupling limit. Therefore, there may be a breakdown in describing the coupled region with a very narrow inter-domain distance in terms of a large $r$. We are speculating that this problem is fundamentally related to the inter-domain charge transfer caused by electron tunneling.

Our description of an EMP crystal in this manuscript looks unrelated to quantum mechanics; however, an essential feature of quantum theory is partly built-in. Suppose that for an EMP molecule, an EMP pulse in the first domain enters the coupled region. In the second domain, at the boundary $x=0$, a pair creation from the vacuum takes place. This 
is a process of the creation of a particle and antiparticle, which is a phenomenon handled by the quantum field theory. We also note that for the diatomic EMP molecule discussed in Sec. III, the energy density may be identified as a potential energy:

$$
H(x)=\left(\begin{array}{ll}
\rho_{1}(x) & \rho_{2}(x)
\end{array}\right)\left(\begin{array}{l}
V_{1}(x) \\
V_{2}(x)
\end{array}\right)=\frac{1}{c_{c h}}\left(\rho_{1}(x) \rho_{2}(x)\right)\left(\begin{array}{cc}
1-\delta & \delta \\
\delta & 1-\delta
\end{array}\right)\left(\begin{array}{l}
\rho_{1}(x) \\
\rho_{2}(x)
\end{array}\right) .
$$

By Eq. (6),$H(x)$ is rewritten as a quadratic form in the charge density variables $\rho_{1}$ and $\rho_{2}$ (or current densities $j_{1}$ and $j_{2}$ ). This is consistent with a quantum mechanical Hamiltonian density, by which a quantum mechanical description of the system is possible based on the $\mathrm{U}(1)$ current algebra. $\underline{\underline{16}}$

There are some possible extensions of the work described in this paper. One is to include the spin (current). For the QHE with $\nu=2$, (dynamical) charge and spin currents coexist at the edge of a single domain. Though it is not evident that the formulation based on a capacitive interaction (between different domains) holds for this case (of different edge channels in the same domain), recent experiments show that this is indeed valid. $\underline{\underline{22}}$ It would also be interesting to include the opposite chirality in the same domain, which is expected for a quantum spin Hall effect. From a theoretical point of view, if the spin degrees of freedom is replaced with pseudospin, such edge plasmon crystal without an external magnetic field is relevant to the plasmons observed in doped carbon nanotubes (albeit with a difference in spatial scales) $\underline{\underline{29}} \underline{\underline{32}}$ Though this appears to be an impossible geometry for EMPs, azimuthal plasmons in doped carbon nanotubes (CNTs) can be treated as a circular current in two dimensions, if the domain is regarded as the cross section of a CNT. This is an issue to which the results of this paper could be applied. We speculate that some discrepancy between theory and experiments found recently $\underline{\underline{33}}$ may be partly resolved by a capacitive coupling between the plasmons.

\section{SUMMARY}

The band structures of EMP crystals (chain, ladder, and honeycomb network) were calculated based on the continuity of the current density with a transfer matrix method. The calculated results are explained by the eigen modes of an EMP molecule composed of two equivalent atoms (domains). We have discussed the effect of a geometrical deformation of 
a chain on the wavenumber in terms of a gauge degree of freedom. We pointed out an interesting similarity between EMP crystals and layered materials (superlattices).

\section{ACKNOWLEDGMENTS}

The author thanks M. Hashisaka and K. Muraki for proposing the problem. The author is also grateful to N. Kumada for his outstanding instruction.

\section{Appendix A: Domains with opposite magnetic field directions}

We show a planar geometry composed of two capacitively coupled domains having opposite magnetic field directions in Fig. 9(a). This configuration of the staggered magnetic field appears to be a little unrealistic. However, as shown in Fig. 9(b), the topologically equivalent configuration in three-dimensions corresponds to a uniform magnetic field, as opposed to that in Fig. 1(c), and thus turns out to be a more realistic. Indeed, when the two domains are merged into a single domain in Fig. 9(b) by setting the inter-domain distance to zero and also $\ell \rightarrow L$, this serves as a model for co-propagating spin-polarized edge channels in a single domain with $\nu=2 \mathrm{QHE} \stackrel{4}{42}^{22}$ The situation is also relevant to a capacitor in an external magnetic field, for which the following analysis would have direct relevance.

(a)

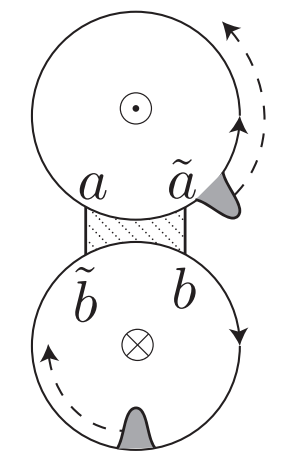

(b)

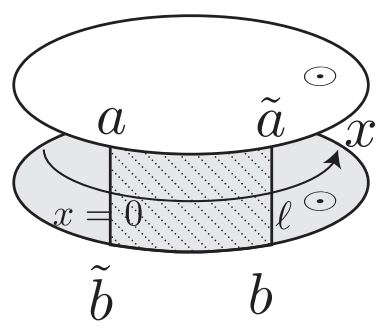

(c)

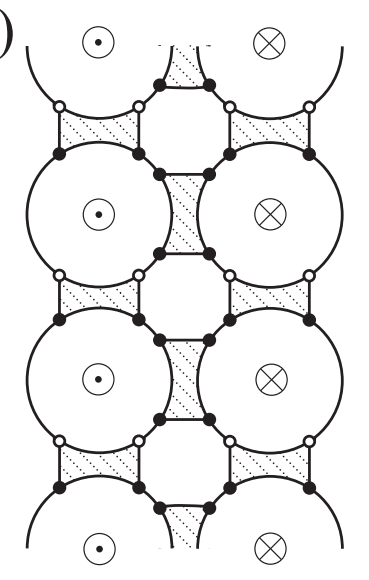

FIG. 9. Geometries of two domains with a staggered magnetic field. The geometry in two dimensions (a) corresponds to (b) in three dimensions. An example of a planar periodic crystals is shown in (c). 
The study of the two domains is rather straightforward. The unique modification that we need to apply is

$$
\left(\begin{array}{l}
j_{1}(x) \\
j_{2}(x)
\end{array}\right)=\sigma_{x y}\left(\begin{array}{ll}
1 & 0 \\
0 & 1
\end{array}\right)\left(\begin{array}{l}
V_{1}(x) \\
V_{2}(x)
\end{array}\right)
$$

instead of Eq. (5). By repeating the same analysis, we have for the coupled region $x \in[0, \ell]$ two modes with the same chirality. The current density is written as

$$
\left(\begin{array}{l}
j_{1}(x) \\
j_{2}(x)
\end{array}\right)=\alpha_{R}\left(\begin{array}{l}
1 \\
1
\end{array}\right) e^{+i \frac{\omega}{v} x}+\beta_{R}\left(\begin{array}{c}
1 \\
-1
\end{array}\right) e^{+i \frac{\omega}{v_{s}} x} .
$$

The velocities of the two modes are $v$ and $v_{s} \equiv(1-2 \delta) v$. Note that the renormalized velocity $v_{s}$ differs from $v_{c}$ by the multiplicative factor of $\sqrt{1-2 \delta}$. Due to the continuity condition of the current density, the current amplitudes at the vertices are related by

$$
\left(\begin{array}{l}
\tilde{a} \\
b
\end{array}\right)=V(\omega)\left(\begin{array}{l}
a \\
\tilde{b}
\end{array}\right)
$$

where the matrix $V$ is defined as

$$
V(\omega) \equiv\left(\begin{array}{cc}
1 & 1 \\
1 & -1
\end{array}\right)\left(\begin{array}{cc}
e^{i \frac{\omega \ell}{v}} & 0 \\
0 & e^{i \frac{\omega \ell}{v_{s}}}
\end{array}\right)\left(\begin{array}{cc}
1 & 1 \\
1 & -1
\end{array}\right)^{-1} .
$$

Applying the phase relations $a=e^{i \frac{\omega(L-\ell)}{v}} \tilde{a}$ and $\tilde{b}=e^{i \frac{\omega(L-\ell)}{v}} b$ to Eq. (A3), we obtain the frequency of a non-bonding state as $\omega=\frac{2 \pi v}{L} n$ and that of a bonding orbital as

$$
\omega=\frac{2 \pi n}{\frac{L-\ell}{v}+\frac{\ell}{v_{s}}} .
$$

For the special case of $\ell=L, \omega=\frac{2 \pi v_{s}}{L} n$. More generally, in order to make the coupled region a limited part of the domain, it is necessary to prepare two domains with different diameters, but such details are ignored here. The $V$ matrix is used to calculate the band structure of a ladder shown in Fig. 9(c), which can be obtained by diagonalizing the matrix $M$ given by

$$
M(\omega)=\left(\begin{array}{ccc}
e^{-i \frac{\omega}{v}\left(\frac{L}{2}-\ell\right)} & 0 & 0 \\
0 & e^{i \frac{\omega s}{v}} V(\omega) e^{i \frac{\omega s}{v}} & 0 \\
0 & 0 & e^{-i \frac{\omega}{v}\left(\frac{L}{2}-\ell\right)}
\end{array}\right)\left(\begin{array}{cc}
T(\omega) & 0 \\
0 & T^{*}(\omega)
\end{array}\right)
$$


It is also useful to define the matrix

$$
T_{s}(\omega) \equiv\left(\begin{array}{cc}
1 & 1 \\
e^{i \frac{\omega \ell}{v}} & e^{i \frac{\omega \ell}{v_{s}}}
\end{array}\right)\left(\begin{array}{cc}
1 & 0 \\
0 & -1
\end{array}\right)\left(\begin{array}{cc}
1 & 1 \\
e^{i \frac{\omega \ell}{v}} & e^{i \frac{\omega \ell}{v_{s}}}
\end{array}\right)^{-1}
$$

that relates the current density of one domain to that of the other domain as

$$
\left(\begin{array}{l}
a \\
\tilde{a}
\end{array}\right)=T_{s}(\omega)\left(\begin{array}{l}
\tilde{b} \\
b
\end{array}\right) .
$$

The $T_{s}$ matrix satisfies $T_{s}^{-1}=T_{s}$ and $\operatorname{det}\left(T_{s}\right)=-1$.

\section{Appendix B: Correspondence to Kronig-Penny model}

The Kronig-Penney model is a model for an electron in a one-dimensional periodic potential. $\stackrel{34}{=}$ In this appendix, we show that an EMP chain bears a remarkable similarity to the electron in a superlattice by studying the model using the method developed for EMPs.

Suppose that the unit cell of the superlattice consists of two layers (A and B) with a potential difference $V$ due to band discontinuity. In the unit cell from $x=-b$ to $a$, the wave function is written as a sum of left and right moving waves as

$$
\psi(x)= \begin{cases}A e^{+i k_{A} x}+\tilde{A} e^{-i k_{A} x} & (0<x<a) \\ B e^{+i k_{B} x}+\tilde{B} e^{-i k_{B} x} & (-b<x<0)\end{cases}
$$

where the wavevector $k_{A}$ and $k_{B}$ are related to the energy eigenvalue $E$ by the Schrödinger equation for the nonrelativistic electron with the effective mass $m$,

$$
\frac{\hbar^{2}}{2 m} k_{A}^{2}=E, \quad \frac{\hbar^{2}}{2 m} k_{B}^{2}+V=E .
$$

Since the wave function and its first derivative with respect to $x$ must be continuous at the boundary between layers $\mathrm{A}$ and $\mathrm{B}$, we obtain the boundary condition at $x=0$ :

$$
\left(\begin{array}{cc}
1 & 1 \\
k_{A} & -k_{A}
\end{array}\right)\left(\begin{array}{c}
A \\
\tilde{A}
\end{array}\right)=\left(\begin{array}{cc}
1 & 1 \\
k_{B} & -k_{B}
\end{array}\right)\left(\begin{array}{c}
B \\
\tilde{B}
\end{array}\right) .
$$

From this, we define two matrices $T_{A B}$ and $T_{B A}$ as follows:

$$
T_{A B} \equiv\left(\begin{array}{cc}
1 & 1 \\
k_{A} & -k_{A}
\end{array}\right)^{-1}\left(\begin{array}{cc}
1 & 1 \\
k_{B} & -k_{B}
\end{array}\right), \quad T_{B A} \equiv T_{A B}^{-1}
$$


so that

$$
\left(\begin{array}{c}
A \\
\tilde{A}
\end{array}\right)=T_{A B}\left(\begin{array}{c}
B \\
\tilde{B}
\end{array}\right), \quad\left(\begin{array}{c}
B \\
\tilde{B}
\end{array}\right)=T_{B A}\left(\begin{array}{c}
A \\
\tilde{A}
\end{array}\right) .
$$

Using these equations, we construct the transfer matrix that relates the wave function at layer $\mathrm{A}$ to that at the nearest neighbor of layer $\mathrm{A}$ as ${ }^{26}$

$$
\left(\begin{array}{c}
A \\
\tilde{A}
\end{array}\right)_{j+1}=T_{A B}\left(\begin{array}{cc}
e^{+i k_{B} b} & 0 \\
0 & e^{-i k_{B} b}
\end{array}\right) T_{B A}\left(\begin{array}{cc}
e^{+i k_{A} a} & 0 \\
0 & e^{-i k_{A} a}
\end{array}\right)\left(\begin{array}{c}
A \\
\tilde{A}
\end{array}\right)_{j}
$$

Finally, applying Bloch's theorem to the diagonalized transfer matrix, we obtain

$$
U\left[T_{A B}\left(\begin{array}{cc}
e^{+i k_{B} b} & 0 \\
0 & e^{-i k_{B} b}
\end{array}\right) T_{B A}\left(\begin{array}{cc}
e^{+i k_{A} a} & 0 \\
0 & e^{-i k_{A} a}
\end{array}\right)\right] U^{\dagger}=\left(\begin{array}{cc}
e^{-i k(a+b)} & 0 \\
0 & e^{+i k(a+b)}
\end{array}\right),
$$

which leads to

$$
\operatorname{tr}\left[T_{A B}\left(\begin{array}{cc}
e^{+i k_{B} b} & 0 \\
0 & e^{-i k_{B} b}
\end{array}\right) T_{B A}\left(\begin{array}{cc}
e^{+i k_{A} a} & 0 \\
0 & e^{-i k_{A} a}
\end{array}\right)\right]=2 \cos [k(a+b)] .
$$

This may be rewritten as the compact form

$$
\cos [k(a+b)]=\cos \left(k_{A} a\right) \cos \left(k_{B} b\right)-\frac{1}{2}\left(\frac{k_{A}}{k_{B}}+\frac{k_{B}}{k_{A}}\right) \sin \left(k_{A} a\right) \sin \left(k_{B} b\right) .
$$

By putting Eq. (B2) into Eq. (Bי), the possible energies that the electron can occupy (or miniband structures $\left.E_{n}(k)\right)$ are obtained as a function of the wavevector $k$.

The mathematical similarity between Eq. (B7) and Eq. (32) becomes more evident for the localized states at layer B with $k_{B}=i / \xi_{B}$, where $\xi_{B}^{-1}=\sqrt{2 m(V-E)} / \hbar(0 \leq E \leq V)$ is the inverse of the decay length. This stems from the fact that the $T(\omega)$ matrix of Eq. (32) [or Eq. (16)] can be reproduced from

$$
T_{A B}\left(\begin{array}{cc}
e^{+i k_{B} b} & 0 \\
0 & e^{-i k_{B} b}
\end{array}\right) T_{B A}
$$

by putting $k_{B}=i / \xi_{B}$ into it as

$$
\frac{1}{-2 i r \sin 2 \phi}\left(\begin{array}{cc}
e^{-2 i \phi}-r^{2} e^{+2 i \phi} & -\left(1-r^{2}\right) \\
1-r^{2} & -\left(e^{+2 i \phi}-r^{2} e^{-2 i \phi}\right)
\end{array}\right),
$$


where $\phi$ and $r$ are defined by $1 / k_{A} \xi_{B} \equiv \tan \phi\left(E=V \cos ^{2} \phi\right)$ and $r \equiv e^{-b / \xi_{B}}$. Thus, if $2 \phi$ and $r$ are identified with $\omega l / v_{c}$ and the EMP coupling strength, respectively, there is a close correspondence between the two systems: for example, studying the EMP which has approximately $\omega_{s}\left(2 \omega_{s}\right)$ is the same thing as studying the electron with $\phi \rightarrow \pi / 2(\pi)$ near the bottom (top) of the potential energy $E \rightarrow 0(E \rightarrow V)$. More explicitly, the above derivation of the $T(\omega)$ matrix originates from the fact that $T_{A B}$ is rewritten as

$$
T_{A B}=\frac{\sqrt{k_{A}^{2}+\xi_{B}^{-2}}}{2 k_{A}}\left(\begin{array}{cc}
e^{-i \phi} & e^{i \phi} \\
e^{i \phi} & e^{-i \phi}
\end{array}\right)=\frac{\sqrt{k_{A}^{2}+\xi_{B}^{-2}}}{2 k_{A}}\left(\begin{array}{cc}
1 & 1 \\
e^{i \phi} & e^{-i \phi}
\end{array}\right)\left(\begin{array}{cc}
e^{-i \phi} & 0 \\
0 & e^{i \phi}
\end{array}\right),
$$

in terms of $\phi$ defined by $k_{A}+i / \xi_{B}=\sqrt{k_{A}^{2}+\xi_{B}^{-2}} e^{i \phi}$ [see Eqs. (B10) and (16)]. This similarity is more than what is naturally expected from the point of view of wave mechanics and suggests the physical phenomena observed in a superlattice may manifest itself in EMP crystals.

By comparing Eq. (B9) with Eq. (33), we find that the superlattice has direct relevance to the EMP chain if we assume that

$$
\begin{aligned}
& \cosh \left(b / \xi_{B}\right)=\frac{1+r^{2}}{2 r} \\
& \frac{1}{k_{A} \xi_{B}}-k_{A} \xi_{B}=2 \cot \left(\frac{\omega \ell}{v_{c}}\right) .
\end{aligned}
$$

The former equation confirms $r=e^{-b / \xi_{B}}$, and a small decay length $\left(\xi_{B} \ll b\right)$ caused by a large $V$ corresponds to a weak coupling. Meanwhile, a large decay length $\left(\xi_{B} \gg b\right)$ corresponds to a strong coupling, which seems to be a reasonable correspondence. The latter equation leads to $1 / k_{A} \xi_{B}=-\tan \left(\frac{\omega \ell}{2 v_{c}}\right)$. The minus sign just appears as a result of the correspondence between Eq. (B7) and Eq. (32) for positive $\omega$.

The correspondence $r=e^{-b / \xi_{B}}$, where $\xi_{B}$ is energy dependent while $r$ is just a coupling constant, is not easy to understand. Such an apparent disagreement may be hidden by taking the limit of $V \rightarrow \infty$ and $b \rightarrow 0$ in such a way that $V b$ is a constant, namely the Dirac delta potential. Though $b / \xi_{B}^{2}$ is a nonzero constant, $b / \xi_{B} \rightarrow 0$, and the potential corresponds to the strong coupling limit $(r \rightarrow 1)$ of an EMP chain. On the other hand, it has been shown that $e^{-b / \xi_{B}}$ can be used as a small parameter in perturbation theory to solve the Kronig-Penney model (and to extract a tight-binding parameter, such as hopping integrals). ${ }^{35}$ Thus, EMP chains may cover the Kronig-Penney model with various unit-cell 
structures. We note that the bound states caused by a negative Dirac delta potential can be studied by taking the $a \rightarrow 0$ limit and changing the origin of the energy as $E \rightarrow E+V$, for the localized states in layer $\mathrm{B}(E<0)$. For the bound states, $k_{A}^{2} a$ is a constant and $k_{B}=i / \xi_{B}$, where $\xi_{B}^{-1}=\sqrt{2 m|E|} / \hbar$. In this case, $r \equiv e^{-b / \xi_{B}}$ may take a general value. In a bipartite model, such a bound state can be doubled in the unit cell, which has been examined from the point of view of toplogically protected edge states ${ }^{36}$ Such a model is more relevant to an EMP chain containing two domains with different domain sizes $L_{1}$ and $L_{2}$ (or different coupling strength $r_{1}$ and $r_{2}$ ) in the unit cell.

* kenichi.sasaki.af@hco.ntt.co.jp

1 S. J. Allen, H. L. Störmer, and J. C. M. Hwang, "Dimensional resonance of the two-dimensional electron gas in selectively doped GaAs/AlGaAs heterostructures," Physical Review B 28, 4875-4877 (1983).

2 D. B. Mast, A. J. Dahm, and A. L. Fetter, "Observation of Bulk and Edge Magnetoplasmons in a Two-Dimensional Electron Fluid," Physical Review Letters 54, 1706-1709 (1985).

3 D. C. Glattli, E. Y. Andrei, G. Deville, J. Poitrenaud, and F. I. B. Williams, "Dynamical Hall Effect in a Two-Dimensional Classical Plasma," Physical Review Letters 54, 1710-1713 (1985).

4 Masayuki Hashisaka and Toshimasa Fujisawa, "Tomonaga-Luttinger-liquid nature of edge excitations in integer quantum Hall edge channels," Reviews in Physics 3, 32-43 (2018).

5 Go Yusa, Wataru Izumida, and Masahiro Hotta, "Quantum energy teleportation in a quantum Hall system," Physical Review A 84, 032336 (2011).

6 R. C. Ashoori, H. L. Stormer, L. N. Pfeiffer, K. W. Baldwin, and K. West, "Edge magnetoplasmons in the time domain," Physical Review B 45, 3894-3897 (1992).

7 V A Volkov and S A Mikhailov, "Edge magnetoplasmons: low-frequency weakly damped excitations in inhomogeneous two-dimensional electron systems," Sov. Phys. JETP 67, 1639 (1988).

8 Sergei A. Mikhailov, "Edge and Inter-edge Magnetoplasmons in Two-dimensional Electron Systems," in Edge excitations of low-dimensional charged systems, edited by Oleg Kirichek (Nova Science Publishers, c2001., Huntington, N.Y, 2001).

9 Ken-ichi Sasaki, Shuichi Murakami, Yasuhiro Tokura, and Hideki Yamamoto, "Determination of intrinsic lifetime of edge magnetoplasmons," Physical Review B 93, 125402 (2016). 
10 V.I. Fal'ko and D.E. Khmel'nitskii, "What if a film conductivity exceeds the speed of light?" Sov. Phys. JETP 68, 1150 (1989).

11 Hugen Yan, Zhiqiang Li, Xuesong Li, Wenjuan Zhu, Phaedon Avouris, and Fengnian Xia, "Infrared spectroscopy of tunable Dirac terahertz magneto-plasmons in graphene." Nano letters 12, 3766-71 (2012).

12 A. H. MacDonald, "Edge states in the fractional-quantum-Hall-effect regime," Physical Review Letters 64, 220-223 (1990).

13 M. Wassermeier, J. Oshinowo, J. P. Kotthaus, A. H. MacDonald, C. T. Foxon, and J. J. Harris, "Edge magnetoplasmons in the fractional-quantum-Hall-effect regime," Physical Review B 41, 10287-10290 (1990).

14 X. G. Wen, "Chiral Luttinger liquid and the edge excitations in the fractional quantum Hall states," Physical Review B 41, 12838-12844 (1990).

15 X. G. Wen, "Electrodynamical properties of gapless edge excitations in the fractional quantum Hall states," Physical Review Letters 64, 2206-2209 (1990).

16 XIAO-GANG WEN, "THEORY OF THE EDGE STATES IN FRACTIONAL QUANTUM HALL EFFECTS," International Journal of Modern Physics B 06, 1711-1762 (1992).

17 C. L. Kane, Matthew P. A. Fisher, and J. Polchinski, "Randomness at the edge: Theory of quantum Hall transport at filling $\nu=2 / 3$," Physical Review Letters 72, 4129-4132 (1994).

18 Zyun F. Ezawa, Quantum Hall Effects: Recent Theoretical and Experimental Developments, Third Edition (World Scientific Publishing Co., 2013) pp. 1-891.

19 N. Kumada, N.-H. Tu, K.-i. Sasaki, T. Ota, M. Hashisaka, S. Sasaki, K. Onomitsu, and K. Muraki, "Suppression of gate screening on edge magnetoplasmons by highly resistive ZnO gate," Physical Review B 101, 205205 (2020).

20 Masayuki Hashisaka, Hiroshi Kamata, Norio Kumada, Kazuhisa Washio, Ryuji Murata, Koji Muraki, and Toshimasa Fujisawa, "Distributed-element circuit model of edge magnetoplasmon transport," Physical Review B 88, 235409 (2013).

21 H. Kamata, N. Kumada, M. Hashisaka, K. Muraki, and T. Fujisawa, "Fractionalized wave packets from an artificial Tomonaga-Luttinger liquid," Nature Nanotechnology 9, 177-181 (2014).

22 M. Hashisaka, N. Hiyama, T. Akiho, K. Muraki, and T. Fujisawa, "Waveform measurement of charge- and spin-density wavepackets in a chiral Tomonaga-Luttinger liquid," Nature Physics 13, 559-562 (2017). 
23 E D Palik and J K Furdyna, "Infrared and microwave magnetoplasma effects in semiconductors," Reports on Progress in Physics 33, 307 (1970).

24 O S Heavens, "Optical properties of thin films," Reports on Progress in Physics 23, 301 (1960).

25 E. E. Mendez, F. Agulló-Rueda, and J. M. Hong, "Stark Localization in GaAs-GaAlAs Superlattices under an Electric Field," Physical Review Letters 60, 2426-2429 (1988).

26 G. Bastard, "Theoretical investigations of superlattice band structure in the envelope-function approximation," Physical Review B 25, 7584-7597 (1982).

27 Masaaki Nakayama, "WANNIER-STARK LOCALIZATION IN SEMICONDUCTOR SUPERLATTICES," Optical Properties of Low-Dimensional Materials , 147-201 (1996).

28 Paul Brasseur, Ngoc Han Tu, Yoshiaki Sekine, Koji Muraki, Masayuki Hashisaka, Toshimasa Fujisawa, and Norio Kumada, "Charge fractionalization in artificial Tomonaga-Luttinger liquids with controlled interaction strength," Physical Review B 96, 081101 (2017).

29 Seiji Uryu, "Numerical study of cross-polarized plasmons in doped carbon nanotubes," Physical Review B 97, 125420 (2018).

30 Daria Satco, Ahmad R. T. Nugraha, M. Shoufie Ukhtary, Daria Kopylova, Albert G. Nasibulin, and Riichiro Saito, "Intersubband plasmon excitations in doped carbon nanotubes," Physical Review B 99, 075403 (2019).

31 Ken-ichi Sasaki and Yasuhiro Tokura, "Theory of a Carbon-Nanotube Polarization Switch," Physical Review Applied 9, 034018 (2018).

32 Kazuhiro Yanagi, Ryotaro Okada, Yota Ichinose, Yohei Yomogida, Fumiya Katsutani, Weilu Gao, and Junichiro Kono, "Intersubband plasmons in the quantum limit in gated and aligned carbon nanotubes," Nature Communications 9, 1121 (2018).

33 Ken-ichi Sasaki, "Dynamical environmental effects lowering the plasmon energy and lifetime in doped carbon nanotubes," Carbon 160, 1-4 (2020).

34 R. De L. Kronig and William George Penney, "Quantum mechanics of electrons in crystal lattices," Proceedings of the Royal Society of London. Series A, Containing Papers of a Mathematical and Physi

35 F. Marsiglio and R. L. Pavelich, "The tight-binding formulation of the Kronig-Penney model," Scientific Reports 2017 7:1 7, 1-8 (2017).

36 Thomas Benjamin Smith and Alessandro Principi, "A bipartite Kronig-Penney model with Dirac-delta potential scatterers," Journal of Physics: Condensed Matter 32, 055502 (2019). 